\title{
Searching for Young Planets with Sparse Aperture Masking
}

\author{
Adam L. Kraus*, Michael J. Ireland ${ }^{\dagger}$, Frantz Martinache** and James P. \\ Lloyd $^{* *}$ \\ ${ }^{*}$ California Institute of Technology \\ ${ }^{\dagger}$ University of Sydney \\ ${ }^{* *}$ Cornell University
}

\begin{abstract}
We describe an ongoing survey to directly detect substellar and planetary companions to nearby young stars. This survey uses adaptive optics and nonredundant aperture-masking interferometry to achieve typical contrast limits of $\Delta K \sim 6$ at the diffraction limit, probing a completely new regime of parameter space. These observations have revealed many new stellar companions, but only a few companions that might be brown dwarfs; this paucity resembles the so-called "brown dwarf desert" that has been observed by RV planet searches. The survey has not detected any extrasolar planets, despite mass detection limits as low as $7 M_{J u p}$, yielding constraints on the population of extrasolar giant planets. Finally, we discuss some of the implications for protoplanetary disk evolution, including potential sources for gap formation and disk dissipation.
\end{abstract}

Keywords: stars:formation, stars:pre-main sequence, planetary systems:formation, planetary systems:protoplanetary disks, binaries:visual

PACS: 95.75.Qr,95.75.Kk,97.10.Bt,97.21.+a,97.80.-d,97.82.Fs,97.82.Jw,98.20.-d

\section{OUR SURVEY}

Our survey encompassed 82 young stars in the nearby OB association Upper Scorpius, spanning a mass range of $\sim 0.5-1.5 M_{\odot}$, which we observed with nonredundant aperture masking (Kraus et al. 2008). Masking resamples the filled telescope aperture into a sparse interferometric array (e.g. Lloyd et al. 2006; Ireland et al. 2008; Figure 1), allowing for precise calibration of the PSF and yielding very deep detection limits. We achieved typical contrasts of $\Delta m \sim 5-6$ at the diffraction limit.

We chose Upper Scorpius because its age ( $~ 5 \mathrm{Myr})$ and distance $(\sim 145 \mathrm{pc})$ make it ideal for detecting young planets (de Zeeuw et al. 1999). The association is very young, so any newly-formed planets should be self-luminous. However, it is also old enough that most of the natal gas and dust have dispersed; the absence of interstellar material leaves stars optically bright, allowing for good AO correction for low-mass cluster members. Most of the protostellar disks have also been cleared by this age (e.g. Carpenter et al. 2006), suggesting that giant planet formation has already reached its conclusion. At the distance of Upper Sco, our survey's separation range (40-300 mas) corresponds to the giant planet regime of our own solar system (5-45 AU). 




FIGURE 1. Schematic diagram of the aperture masking apparatus. The telescope pupil is reimaged at the imager's Lyot stop, transforming the image in the focal plane into an interferogram. A fourier transform then yields the visibilities, from which closure phases between triangles (colored circles) can be measured.
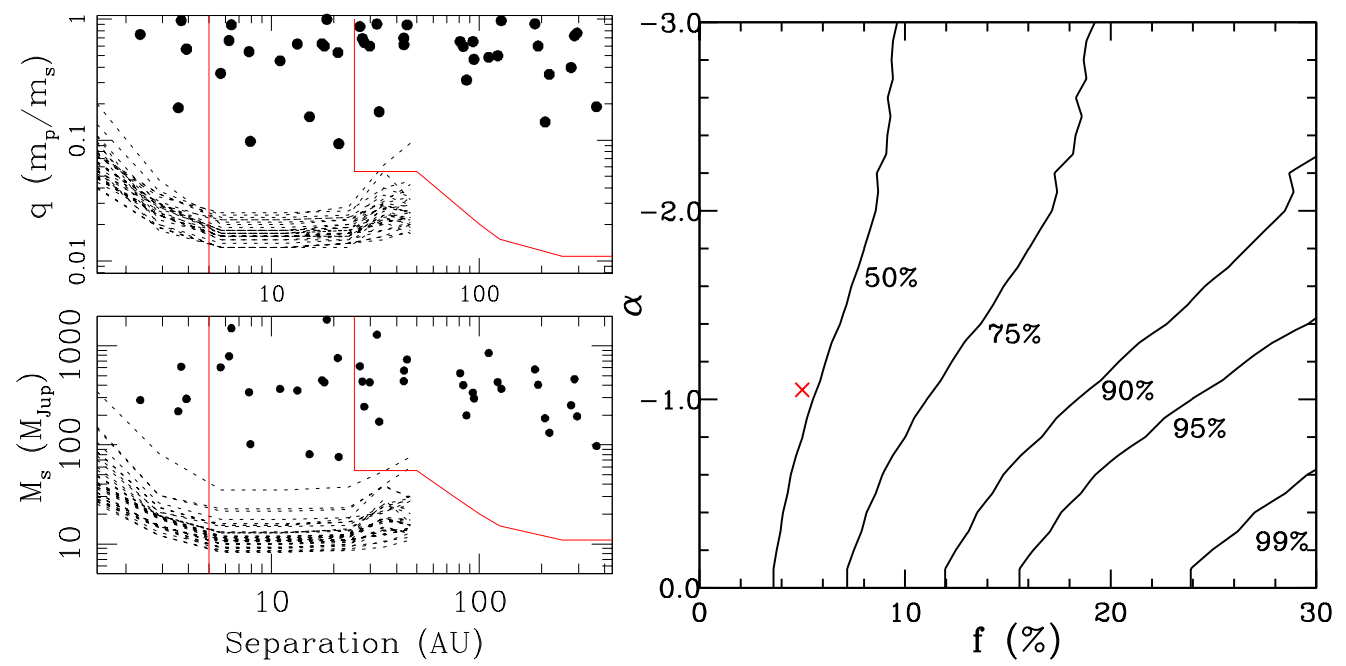

FIGURE 2. Left: Detection limits at Keck for our Upper Sco targets in terms of mass ratio $q$ (top) and secondary mass (bottom). The dashed lines denote the $99.5 \%$ detection limits for all of our sample members, while the filled circles show all binary companions in our survey. Right: Joint confidence intervals for the frequency of Jovian companions $f$ and the planetary mass function power law exponent $\alpha$ as determined from our detection limits and Monte Carlo simulations. The red cross shows the bestestimated values from RV surveys ( $f \sim 5 \%$ and $\alpha \sim-1.0)$.

\section{EXTRASOLAR PLANETS}

As we show in Figure 2 (left), our survey's mass detection limits encompass the entire brown dwarf mass range and the top of the planetary mass regime, while the separation regime complements the typical limits for $\mathrm{RV}$ monitoring $(<5 \mathrm{AU})$ and coronagraphic 

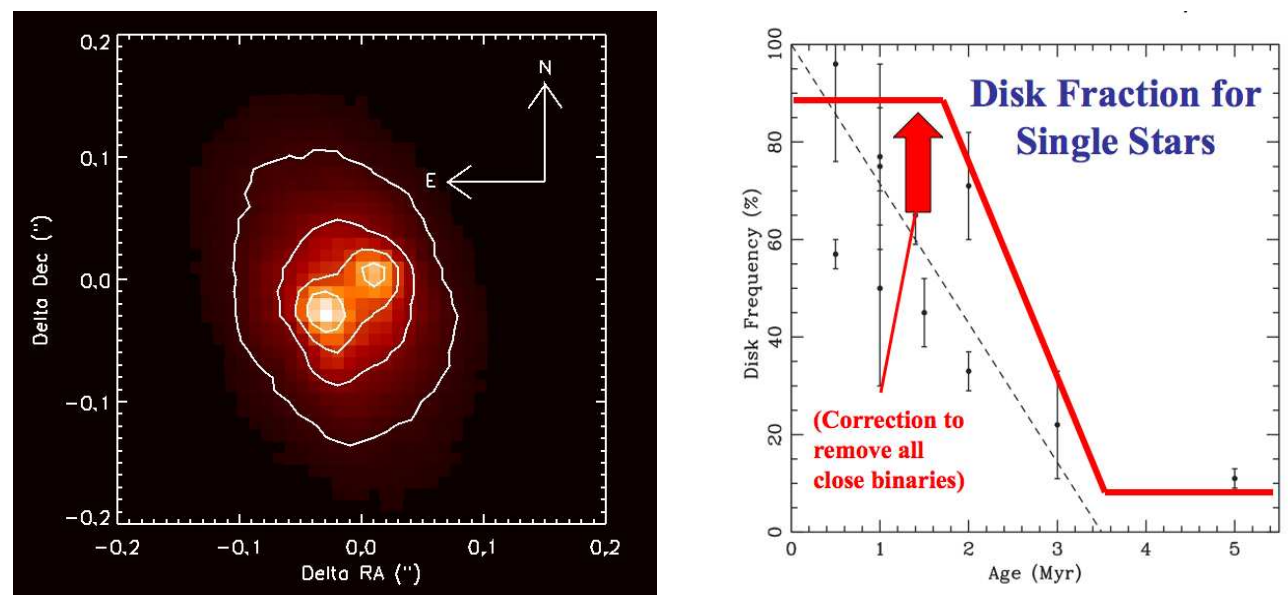

FIGURE 3. Left: $\mathrm{CoKu} \mathrm{Tau} / 4 \mathrm{AB}$, a binary system that was previously thought to be a transitional disk host. Right: Disk frequency as a function of age for several stellar populations (e.g. Haisch et al. 2001, Hillenbrand 2005), plus a schematic view of the correction needed for unresolved binary systems if our Taurus results are representative of all star-forming regions.

imaging ( $>25 \mathrm{AU}$ ). We found numerous binary companions, but there is a paucity of companions with masses $<75 M_{J u p}$ that resembles the so-called "brown dwarf desert".

Our survey did not detect any companions in the planetary-mass regime, so we used Monte Carlo simulations to determine our constraints on the underlying planetary population. We specifically modeled the population in terms of a frequency $f$ (for planets with mass $>1 M_{J u p}$ ), a power-law mass function with exponent $\alpha$, and a power-law separation distribution with exponent $\beta$. In Figure 2 (right), we show the resulting joint constraints on $f$ and $\alpha$, where the red cross shows the most likely values estimated by RV surveys. Our nondetection is only significant at the $50 \%$ level, so there is no disagreement with those canonical values. However, we can rule out the joint occurrance of a high frequency with a steeper mass function or separation distribution. We also note that these simulations predict a $50 \%$ chance of finding a planet in our survey, emphasizing the need to expand the sample.

\section{THE ROLE OF MULTIPLICITY IN PROTOPLANETARY DISK EVOLUTION}

We are extending our survey into Taurus-Auriga and Ophiuchus, where our highestpriority targets are the so-called "transitional disk" hosts: young stars whose protoplanetary disks possess significant amounts of cold dust at large radii, but a paucity of warm dust at smaller radii. This paucity has been interpreted as a sign of recent or ongoing planet formation in the inner regions of these disks, so these stars could offer the best probability of directly observing young planets.

We did not detect any close companions to GM Aur, LkCa15, or UX Tau A, suggesting that if newly-formed are clearing their disks, those planets must be less massive than 8-10 $M_{\text {Jup }}$. However, we discovered that $\mathrm{CoKu} \mathrm{Tau} / 4$ is an equal-mass binary system 


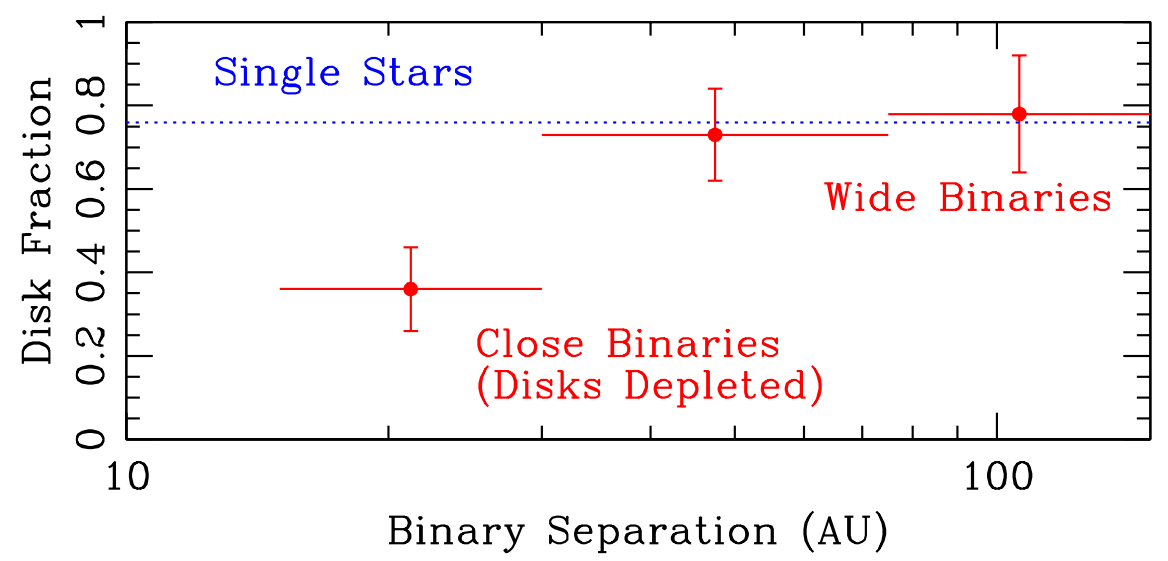

FIGURE 4. Disk fraction as a function of separation for all binary systems in Taurus (red filled circles). The disk fraction for all apparently single stars is indicated by a blue dashed line.

with a projected separation of $\sim 8$ AU (Figure 3, left; Ireland \& Kraus 2008); given an inner disk radius of $\sim 10-15 \mathrm{AU}$, then the "transitional" disk must actually be a (quasistatic) circumbinary disk that is cleared by tidal resonances from the binary system.

Overall, we have found that the frequency of close ( $<20 \mathrm{AU}$ ) systems is $\sim 20 \%$, so this raises an important question: why don't we see dozens of circumbinary "transitional disks" like CoKu Tau/4? As we show in Figure 4, many of the closest binary systems are found preferentially among the WTTS population. The disk fraction drops sharply at separations of $<30 \mathrm{AU}$; single stars and wide binary systems have disk fractions of $\sim 0.8$, while 15-30 AU binary systems have disk fractions of $\sim 0.35$. Our preliminary results suggest that the disk fraction is even lower for closer binary systems. The implication is that binary systems clear their disks much more quickly than single stars.

This trend suggests that previous studies of disk evolution might have suffered from a significant systematic bias. Indeed, if we remove our new binary systems from disk dissipation studies of supposedly single stars (Figure 3, right; e.g. Haisch et al. 2001, Hillenbrand et al. 2005), it appears that the disk frequency for single stars might be as high as $90 \%$ at ages of 1-2 Myr. Our results indicate that little disk evolution occurs at earlier ages, and therefore that most single stars have at least 1-2 Myr within which to form giant planets. Conversely, circumbinary disks have a much shorter interval within which to form planets.

\section{REFERENCES}

1. Carpenter, J., Mamajek, E., Hillenbrand, L., \& Meyer, M. 2006, ApJ, 651, 49

2. de Zeeuw, P., Hoogerwerf, R., de Bruijne, J., Brown, A., \& Blaauw, A. 1999, AJ, 117, 354

3. Haisch, K., Lada, E., \& Lada, C. 2001, ApJ, 553, 153

4. Hillenbrand, L. 2005, in A Decade of Discovery: Planets Around Other Stars, ed. M. Livio (Baltimore: STScI), preprint (astro-ph/0511083)

5. Ireland, M. \& Kraus, A. 2008, ApJ, 678, L59

6. Ireland, M., Kraus, A., Martinache, F., Lloyd, J., \& Tuthill, P. 2008, ApJ, 678, 463

7. Kraus, A., Ireland, M., Martinache, F., \& Lloyd, J. 2008, ApJ, 679, 762

8. Lloyd, J. et al. 2006, ApJ, 650, 131 